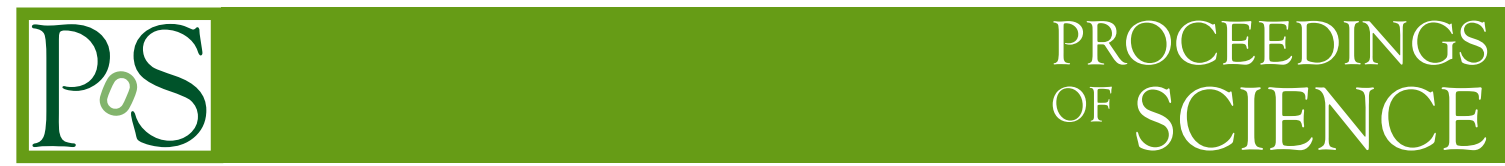

\title{
Studies of WW and WZ production at CDF
}

\author{
Viviana Cavaliere ${ }^{* \dagger}$ \\ University of Siena - Via Roma 56, Siena (Italy) \\ INFN Pisa - Largo B. Pontecorvo 3, Pisa (Italy) \\ E-mail: viviana.cavaliere@pi.infn.it
}

\begin{abstract}
Diboson production in $p \bar{p}$ collisions at $1.96 \mathrm{TeV}$ are studied in samples of $\sim 3-6 \mathrm{fb}^{-1}$ of data using leptons, jets and missing $E_{T}$. Fully leptonic decays as well as semi-leptonic decays are measured since it is important to investigate various signatures as associated production of Higgs bosons is topologically similar. Measured production cross sections are in good agreement with Standard Model predictions and the limits on the anomalous triple gauge boson couplings are competitive with the ones measured by experiments at the LEP.
\end{abstract}

35th International Conference of High Energy Physics - ICHEP2010,

July 22-28, 2010

Paris France

\footnotetext{
${ }^{*}$ Speaker.

$\dagger$ for the CDF Collaboration
} 


\section{Introduction}

Diboson production is of great interest because it provides unique opportunity to test the Standard Model (SM) at the TeV scale, it constitutes a very important background to Higgs and SUSY searches and it is a probe to new physics through deviations of Triple Gauge Couplings (TGCs) from SM predictions. In this paper we examine diboson production $(W W, W Z, Z Z)$ in their leptonic and semi-leptonic final states.

\section{Diboson production in leptonic final state}

These final states are characterized by low branching ratio and clean yields. All the analysis use improved lepton definitions to increase the acceptance.

\subsection{WW cross section and TGC}

We measured the $W W$ production cross section in the final state with two charged leptons ( $e$ or $\mu$ ) and missing energy $\left(E_{T}^{\prime}\right)$ using $3.6 \mathrm{fb}^{-1}$ of integrated luminosity [1]. The $W W$ cross section is extracted using a binned fit to the likelihood ratio distribution formed from Matrix Element probabilities of signal and background as estimated from Monte Carlo simulation (MC). The measured cross section is $12.1 \pm 0.9$ (stat. $)_{-1.4}^{+1.6}$ (syst.) pb, that is in good agreement with the SM prediction and represents the most precise measurement up to date.

The study of the $p_{T}$ distribution allows to set limits on TGCs. Using the HISZ parameterization [2] we measured the $95 \% \mathrm{CL}$ intervals to be: $-0.17<\lambda^{\mathrm{Z}}<0.17,-0.26<\Delta g_{1}^{\mathrm{Z}}<0.35$, and $-0.68<\Delta \kappa^{\gamma}<0.77$, where $\Delta g_{1}^{Z}$ and $\Delta \kappa^{\gamma}$ are the deviations of the parameters from the SM values (that are $\lambda^{\mathrm{Z}}=0, g_{1}^{\mathrm{Z}}=\kappa^{\gamma}=1$ ).

\subsection{WZ cross section}

The $W Z$ production cross section has been measured in the final state with three leptons ( $e$ or $\mu$ ) plus $E_{T}^{\prime}$ in $6.0 \mathrm{fb}^{-1}$ of integrated luminosity [3]. A dedicated lepton selection has been performed to gain efficiency on the signal reconstruction. The ratio between the $W Z \rightarrow 3 \ell v$ and $Z \rightarrow \ell \ell$ cross sections is measured to reduce the systematic uncertainties. Using as input the theoretical value of the $Z \rightarrow \ell \ell$ cross section we extract $\sigma(W Z)=4.1 \pm 0.7$ (stat.+syst.) pb, compatible with the SM prediction.

\section{Diboson production in semi-leptonic final state}

Diboson production in final state with jets is much more challenging due to large background from $V+$ jets $(V=Z, W)$. Those events are interesting because their topology is similar to events where a Higgs boson is produced in association with a $W$ or a $Z$, allowing diboson measurements to provide an important step towards possible future measurement of Higgs production.

3.1 $V V \rightarrow E_{T}+$ jets

We measured the diboson cross section using events with large $E_{T}(>60 \mathrm{GeV})$ and two jets with $E_{T}$ above $25 \mathrm{GeV}$ [4]. Due to limited energy resolution we cannot distinguish between $W W$, 


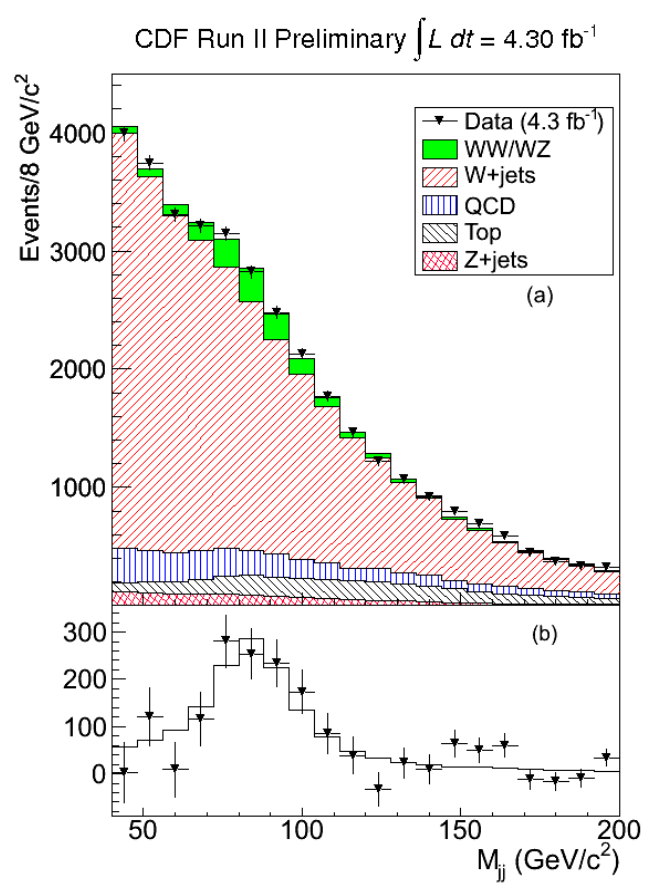

Figure 1: Dijet invariant mass distribution of reconstructed $\mathrm{W} / \mathrm{Z} \rightarrow j j$ candidates compared to the fitted signal and background components.
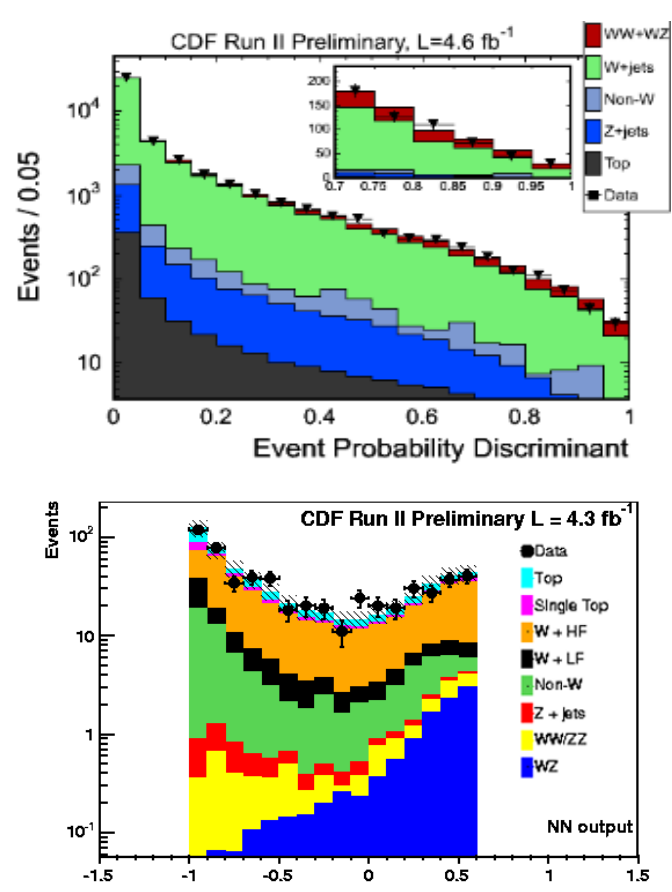

Figure 2: Top: Observed EPD distribution superimposed on expected distribution from simulation. Bottom: Fitted NN output for $W Z$ search.

$W Z$ and $Z Z$ events so what we measure is really a sum of all these processes in our selection window. No cut on number of leptons in the event is performed therefore we are also sensitive to lepton decays of the gauge bosons. The QCD contribution, which is large in this channel, is heavily suppressed through novel algorithms related to $E_{T}$ significance. We extract the signal from the background using the invariant mass distribution of the two jets in the event. The extraction of the signal does not use the theoretical calculation of the $\mathrm{V}+\mathrm{jets}$ cross section and its invariant mass shape is cross checked with $\gamma+$ jets events from the data, hence considerably reducing the systematic uncertainty on the shape of this main background. The significance of the production is $5.3 \sigma$ and the cross section is measured to be $\sigma(p \bar{p} \rightarrow V V+X)=18.0 \pm 2.8$ (stat.) \pm 2.4 (syst.) \pm 1.1 (lum.) pb, in good agreement with the SM expectations.

\section{2 $W W / W Z \rightarrow \ell v+$ jets}

Two different approaches are used to measure the cross section in this channel. They both select signal samples requiring one identified lepton with $E_{T}>20 \mathrm{GeV}$, significant $E_{T}>25 \mathrm{GeV}$ and jets.

The first one uses a data sample corresponding to approximately $4.3 \mathrm{fb}^{-1}$ of integrated luminosity to reconstruct $W W / W Z$ events [5]. The diboson signal is extracted from the background using a $\chi^{2}$ fit of the invariant mass distribution, $M_{j j}$, of the two leading jet separately for the electron and muon samples. This simple method allows to search for a signal peak over a smooth background. The fit is performed in the $M_{j j}$ region from 28 to $200 \mathrm{GeV} / \mathrm{c}^{2}$ and estimates the fractions of signal, QCD and EWK backgrounds using $M_{j j}$ templates obtained from the CDF full simulation 
(signal and EWK) and data (QCD). The total $W+$ jets contribution is a free parameter of the fit while the normalization of the other background is Gaussian constrained to either their expected cross section ( $t \bar{t}$, single top), the measured cross section ( $Z+$ jetS) or from a separate fit to the $E_{T}$ distribution to the data (QCD normalization). The fit is shown in Fig. 1 and corresponds to a statistical significance of $5.2 \sigma(5.1 \sigma$ expected). We measure $\sigma(W W / W Z)=18.1 \pm 3.3$ (stat. $) \pm 2.5$ (syst.).

The other approach uses $4.6 \mathrm{fb}^{-1}$ and takes advantage of a multivariate technique to exploit all the information in the event [6]. Event probability densities are calculated under the signal and background hypotheses using a set of measured variables of each event (the 4-vectors of the lepton and the two jets). Then the probabilities are used to construct a discriminant variable for each event, referred to as the Event Probability Discriminant, or EPD. To quantify the $W W+W Z$ content in the data, a binned maximum likelihood fit is performed (see Fig. 2 top). The background normalizations (except for $W+$ jets that is a free parameter) are Gaussian constrained in the fit. Pseudo-experiments are carried out to determine the significance of the excess that is found to be $5.4 \sigma\left(5.1 \sigma\right.$ expected). The measured cross section is $16.5_{3.0}^{+3.3} \mathrm{pb}$, compatible with the previous one and with the SM prediction.

\section{4. $W Z \rightarrow \ell v b \bar{b}$}

After the observation of diboson in the semileptonic final state, the mandatory step toward Higgs searches is the search for $W Z \rightarrow \ell v b \bar{b}$ [7]. Events are selected requiring one lepton, $E_{T}>20$ $\mathrm{GeV}$ and two jets. The two jets are identified to come from a $b$ quark using a Neural Net (NN) algorithm that uses both the lifetime information as well as the lepton information. Discrimination between the $W Z$ signal and the comparatively large backgrounds is improved through the use of a different NN (see Fig. 2 bottom). The analysis sets a limit based on $4.3 \mathrm{fb}^{-1}$ of $\sigma_{o b s}<3.9 \sigma_{S M}$ at 95\% CL.

\section{5. $Z W+Z Z \rightarrow \ell \ell+j e t s$}

A search for $Z W$ and $Z Z$ production in leptons+ jets final state is also carried out based on $4.8 \mathrm{fb}^{-1}$. This channel is dominated by $Z+j$ ets production. A Jet likelihood ratio is formed to discriminate between jets coming from quarks and jets coming from gluons. Based on the events that pass a cut imposed on the likelihood ratio, a $\mathrm{NN}$ discriminat is formed and fitted to a linear combination of the backgrounds. We set as upper limit on the cross section $\sigma_{Z W+Z Z}<22 \mathrm{pb}$ where the theoretical prediction is $7.5 \mathrm{pb}$.

\section{Conclusion}

The recent results in diboson production at the Tevatron have been presented in both leptonic and semi-leptonic final states. Measured cross sections and TGCs for these processes are in agreement with the SM predictions. CDF is now observing processes with cross sections less than a factor 10 larger than the SM Higgs cross section, and on the way of studying the diboson production with $b$ jets in the final state. 


\section{References}

[1] CDF Collaboration, Phys.Rev.Lett. 104: 201801, 2010

[2] K.Hagiwara, S. Ishihara, R. Szalapski and D. Zeppenfeld, Phys. Rev. D 48, 2182 (1993).

[3] CDF Collaboration, http://www-cdf.fnal.gov/physics/ewk/2010/WZ_ZZ/

[4] CDF Collaboration, Phys.Rev.Lett. 103: 091803, 2009

[5] CDF Collaboration,

http://wWw-cdf.fnal.gov/physics/ewk/2010/WW_WZ/index.html

[6] CDF Collaboration, arXiv:1008.4404v1

[7] CDF Collaboration,

http://www-cdf.fnal.gov/physics/new/hdg//Results_files/results/wzlnubb_100521/10044_blessed.hËml) 